\title{
Thermal Coefficient of Redox Potential of Alkali Metals
}

\author{
Yuya Fukuzumi ${ }^{1}$, Yoyo Hinuma ${ }^{2}$, and Yutaka Moritomo H,3,4* $^{\text {* }}$ \\ ${ }^{1}$ Graduate School of Pure and Applied Science, University of Tsukuba, Tsukuba 305-8571, \\ Japan \\ ${ }^{2}$ Center for Frontier Science, Chiba University, Chiba 263-8522 Japan \\ ${ }^{3}$ Faculty of Pure and Applied Science, University of Tsukuba, Tsukuba 305-8571, Japan \\ ${ }^{4}$ Tsukuba Research Center for Energy Materials Science (TREMS), University of Tsukuba, \\ Tsukuba 305-8571, Japan
}

The thermal coefficient $(\alpha)$ of redox potential $(V)$ is a significant physical quantity that converts the thermal energy into electric energy. In this short note, we carefully determined $\alpha$ of alkali metals $(A=\mathrm{Li}$ and $\mathrm{Na})$ against electrolyte solution. The obtained $\alpha$ is much larger than that expected from the specific heat $\left(C_{\mathrm{p}}^{A}\right)$ of solid $A$ and depends on electrolyte solution. These observations indicate that the solvent has significant effect on $\alpha$.

The thermal coefficient ( $\alpha \equiv \frac{\partial V}{\partial T} ; T$ is temperature) of redox potential $(V)$ is a significant physical quantities for efficient energy conversion of the waste heat near room temperature. Actually, several researchers ${ }^{1-4)}$ reported extraction of electric energy from thermal cycles with high thermal efficiency $\eta(=1-2 \%)$. These thermocells consist of anode, electrolyte solution, and cathode. Important point is that two kinds of redox materials with different $\alpha$ are used as anode and cathode. Then, the heating/cooling of the thermocell changes the relative voltage $\left(V_{\text {cell }}\right)$ between the anode and cathode. The thermocell is in a sharp contrast with the semiconductor-based thermoelectric devices, in which thermal gradient is indispensable. Lee et.al ${ }^{1)}$ and Yang et. al. ${ }^{2)}$ demonstrated the energy conversion with use of redoxable ions, ${ }^{5)}$ e.g., $\left[\mathrm{Fe}(\mathrm{CN})_{6}\right]^{3+} /\left[\mathrm{Fe}(\mathrm{CN})_{6}\right]^{4+}$ and $\mathrm{Cu}^{+} / \mathrm{Cu}^{2+}$, as anode and Prussian blue analogues (PBA) as cathode. Their thermocells, however, are bulky and heavy due to the electrolyte containing redoxable ions. Shibata et al. ${ }^{3)}$ overcome the drawback by fabricating a battery-type thermocell

*moritomo.yutaka.gf@u.tsukuba.ac.jp 
Table I. Thermal coefficient $(\alpha)$ of redox potential $(V)$ of alkali metals $(A)$. EC, DEC, GBL, PC, and DME represent ethylene carbonate, diethyl carbonate, $\gamma$-buthyro-lactone, propylene carbonate, and 1,2dimethoxyethane, respectively. Solvent contribution $\left(\alpha-\frac{1}{e} C_{\mathrm{p}}^{A}\right.$, where $\frac{1}{e} C_{\mathrm{p}}^{A}$ is the specific heat of the solid $\left.A\right)$ is also listed.

\begin{tabular}{rrrrr}
\hline$A$ & solvent & electrolyte & $\alpha(\mathrm{mV} / \mathrm{K})$ & $\alpha-\frac{1}{e} C_{\mathrm{p}}^{A}(\mathrm{mV} / \mathrm{K})$ \\
\hline $\mathrm{Li}$ & $\mathrm{EC} / \mathrm{DEC}$ & $1 \mathrm{M} \mathrm{LiClO}_{4}$ & 0.76 & 0.50 \\
$\mathrm{Li}$ & $\mathrm{GBL}$ & $1 \mathrm{M} \mathrm{LiClO}_{4}$ & 0.88 & 0.62 \\
\hline $\mathrm{Na}$ & $\mathrm{EC} / \mathrm{DEC}$ & $1 \mathrm{M} \mathrm{NaClO}_{4}$ & 0.77 & 0.47 \\
$\mathrm{Na}$ & $\mathrm{GBL}$ & $1 \mathrm{M} \mathrm{NaClO}_{4}$ & 0.73 & 0.43 \\
$\mathrm{Na}$ & $\mathrm{PC}$ & $1 \mathrm{M} \mathrm{NaClO}_{4}$ & 0.71 & 0.41 \\
$\mathrm{Na}$ & $\mathrm{DME}$ & $1 \mathrm{M} \mathrm{NaClO}_{4}$ & 1.70 & 1.40 \\
\hline
\end{tabular}

with use of two kinds of PBAs with different $\alpha$ as anode and cathode. To explore and design high- $\alpha$ metrical for energy conversion, microscopic comprehension of $\alpha$ is indispensable.

Here, let us consider $\alpha$ of the simplest case, $i$. e., redox process of alkali metal $(A)$ in solution: $A$ (solid) $\rightarrow A^{+}$(in solution) $+e^{-}$(in solid $A$ ). In the thermodynamical point of view, $\alpha$ is equivalent to $\frac{1}{e} \frac{\partial \Delta H}{\partial T}$, where $e$ and $\Delta H$ is the elementary charge $(>0)$ and the difference between enthalpies $(H)$ before reaction and that after reaction, respectively. In the present case, $\Delta H$ consists of three parts, i. e., (a) removal of $A$ from the solid $A$, (b) addition of $A^{+}$to the solution, and (c) addition of $e^{-}$to the solid $A$. We note that $\frac{\partial H}{\partial T}$ is equivalent to specific heat $\left(C_{\mathrm{p}}\right)$ at constant pressure. As a result, $\alpha$ can be separated into changes of three specific heats, which are (a) $C_{\mathrm{p}}^{A}$ of the solid $A$ arising from increase of $A$, (b) $C_{\mathrm{p}}^{\mathrm{s}}$ of the solution, which could change significantly because of desolvation in addition to decrease of $A^{+}$, and (c) electron specific heat $\left(C_{\mathrm{p}}^{\mathrm{e}}\right)$ in solid $A$ arising from decrease of $\mathrm{e}^{-}$. Among them, the third component is negligible due to the Fermi degeneracy. Actually, $C_{\mathrm{p}}^{\mathrm{e}}$ of $\mathrm{Li}(\mathrm{Na})$ is estimated to be 5 (4) $\mu \mathrm{eV} / \mathrm{K}$ at $300 \mathrm{~K}$ with use of electronic specific heat coefficient $\gamma\left[=1.7(1.4) \times 10^{-5} \mathrm{eV} / \mathrm{K}^{2}\right]$.

In the present note, we carefully determined $\alpha$ of the most prototypical alkali metals ( $A$ $=\mathrm{Li}$ and $\mathrm{Na}$ ) against electrolyte solution. The obtained $\alpha$ is much larger than $\frac{1}{e} C_{\mathrm{p}}^{A}$ of the solid $A$ and depends on electrolyte solution. These observations indicate that the solvent has significant effect on $\alpha$.

$\alpha$ of $\mathrm{Li}$ and $\mathrm{Na}$ were carefully determined with use of specially-designed thermocell (Fig. 1). The electrolyte was filled in a $\phi 7.3 \mathrm{~mm}$ polytetrafluoroethylen (PTFE) tube. The both ends are sealed with the Al pedestals, on which SUS or Al foils are attached as anode and cathode The two electrodes were placed strictly parallel with a distance of $2 \mathrm{~mm}$, which causes pseudo one-dimensional thermal gradient within the electrolyte solution. The tem- 


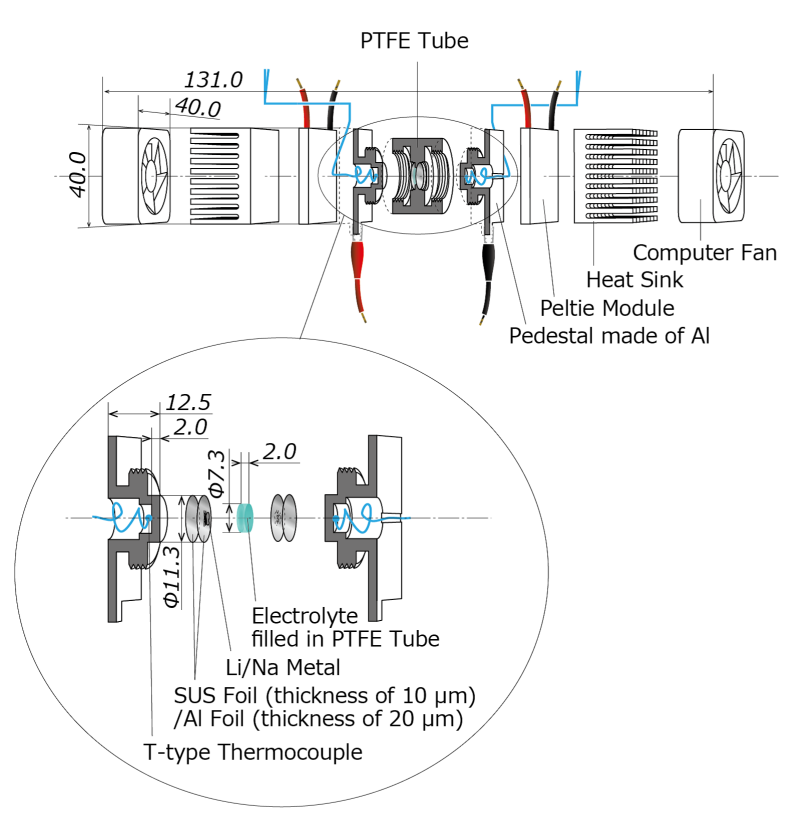

Fig. 1. (Color online) Illustration of specially-designed thermocell. Scales are in mm units.

peratures of anode $\left(T_{\text {anode }}\right)$ and cathode $\left(T_{\text {cathode }}\right)$ are monitored with T-type thermocouples. The thermocouples are attached at the pedestals at a distance of $2 \mathrm{~mm}$ from the electrodeelectrolyte interfaces. $T_{\text {anode }}$ and $T_{\text {cathode }}$ are independently controlled with Peltier modules attached at the bottom of the pedestals. In the measurements, small pieces of the same $A$ were attached at the anode and cathode foils. The difference $\left(\Delta V=V_{\text {cathode }}-V_{\text {anode }}\right)$ in the redox potential of $A$ was carefully measured against $T_{\text {cathode }}$ with fixing $T_{\text {anode }}$ at $295 \mathrm{~K}$ or $292 \mathrm{~K}$. We note that the Seebeck coefficient $(S=-2 \mu \mathrm{V} / \mathrm{K})$ of the Al pedestal is negligible as compared with the observed $\alpha(\geq 0.7 \mathrm{mV} / \mathrm{K})$. The temperature difference $\left(\Delta T=T_{\text {cathode }}\right.$ $T_{\text {anode }}$ ) between the electrodes was less than $10 \mathrm{~K}$. We investigated $\alpha$ in six combinations of $A$ and electrolyte solutions, as listed in Table I. All the measurements were performed without current flow to prevent solid electrolyte interface $\left(\mathrm{SEI}^{6)}\right)$ from forming on the $A$ surface.

Figure 2(a) shows $\Delta V$ of Li against $\Delta T$ with two kinds of electrolyte solutions, $i . e$, EC/DEC and GBL. In both the cases, $\Delta V$ linearly increases with $\Delta T$. The $\alpha$ values were evaluated by least-squares fittings, as indicated by solid straight lines. In Table I, we summarize $\alpha$ against $A$ and electrolyte solutions. $\alpha$ of Li slightly depends on the electrolyte solutions; $\alpha$ is 0.76 and $0.88 \mathrm{mV} / \mathrm{K}$ in EC / DEC and GBL, respectively. In addition, $\alpha$ is much larger than $\frac{1}{e} C_{\mathrm{p}}^{\mathrm{Li}}\left[=0.26 \mathrm{mV} / \mathrm{K},{ }^{7)}\right.$ broken straight line $]$. This indicates that the solvent effect on $\alpha$ is 

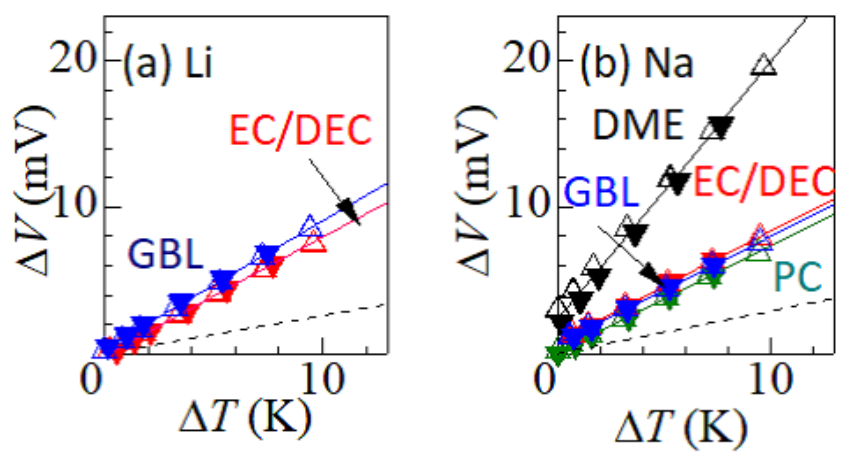

Fig. 2. (Color online) Difference $\left(\Delta V=V_{\text {cathode }}-V_{\text {anode }}\right)$ in the redox potentials of alkali metal $(A)$ against difference $\left(\Delta T=T_{\text {cathode }}-T_{\text {anode }}\right)$ in temperatures between cathode and anode: (a) Li and (b) Na. Error bars ( $\leq$ $0.01 \mathrm{mV}$ ) are within the symbol size. Solid straight lines are results of least-squares fittings. Open and closed simples represent that the data are obtained in the heating and cooling runs, respectively. Broken straight lines show $\frac{1}{e} C_{\mathrm{p}}^{A}$ of solid $A$. EC, DEC, GBL, PC, and DME represent ethylene carbonate, diethyl carbonate, $\gamma$-buthyrolactone, propylene carbonate, and 1,2-dimethoxyethane, respectively.

important and can never be neglected.

Figure 2(b) shows $\Delta V$ of $\mathrm{Na}$ against $\Delta T$ with four kinds of electrolyte solutions, i. e., EC / DEC, GBL, PC and DME. In EC/DEC, GB, and PC, $\alpha$ of Na scarcely depends on the electrolyte solutions; $\alpha$ is $0.77,0.73$, and $0.71 \mathrm{mV} / \mathrm{K}$ in EC / DEC, GBL, and PC, respectively. However, $\alpha$ is much larger than $\frac{1}{e} C_{\mathrm{p}}^{\mathrm{Na}}\left[=0.30 \mathrm{mV} / \mathrm{K},{ }^{7)}\right.$ broken straight line $]$. We emphasize that $\alpha(=1.70 \mathrm{mV} / \mathrm{K})$ of $\mathrm{Na}$ in DME is significantly larger than those in other solutions. Thus, the magnitude of the solvent effect shows significant solvent dependence. We note that DME has no carbonyl group in sharp contrast with the other solvents. This implies that the observed solvent dependence is ascribed to existence/absence of carbonyl group.

Here, let us discuss the microscopic origin of the solvent effect on $\alpha$, i.e., the variation of $C_{\mathrm{p}}^{\mathrm{s}}$ of the solution when one $A^{+}$is dissolved. If one $A^{+}$is dissolved in solvent, the surrounding solvent molecules are forcedly aligned with negatively polarized moieties towards $A^{+}$. Such an alignment of the solvent molecules, or solvation effect, decreases the number of degrees of freedom other than internal vibration in the solution. The reduction of the external vibrational modes suppresses $C_{\mathrm{p}}^{\mathrm{s}}$, and therefore increased $\alpha$. This argument is consistent with the experimental observation: the solvent contribution $\left(=\alpha-\frac{1}{e} C_{\mathrm{p}}^{A}\right.$; Table I) is positive In addition, in the case of EC / DEC and GBL, the solvent contribution is larger for Li as compared with that for $\mathrm{Na}$. This is reasonable because the solvation effect is stronger in the smaller $\mathrm{Li}^{+}$.

Finally, let us briefly comment on the above-discussed solvent effect on the actual ther- 
mocell. In the battery-type thermocell, ${ }^{3)}$ the cathode and anode are in contact with the same electrolyte solution and at the same temperature $\left(T_{\text {cathode }}=T_{\text {anode }}\right)$. In this case, the solvent effect at cathode completely cancel that at anode, because one $A^{+}$is dissolved in solvent when one $A$ precipitates at another electrode. In other words, $\frac{\partial V_{\text {cell }}}{\partial T}\left(=\alpha_{\text {cathode }}-\alpha_{\text {anode }}\right.$; where $\alpha_{\text {cathode }}$ and $\alpha_{\text {anode }}$ are the thermal coefficients of redox potential at cathode and anode, respectively) is free from the solvent effect. We, however, may positively utilize the solvent effect in thermoelectric conversion with use of a thermocell, in which the cathode and anode are in contact with the different electrolyte solution.

In summary, $\alpha$ of $\mathrm{Li}$ and $\mathrm{Na}$ in several electrolyte solutions are carefully determined. The obtained $\alpha$ is much larger than $\frac{1}{e} C_{\mathrm{p}}^{A}$ and depends on electrolyte solution. These observations indicate that the solvent effect on $\alpha$ is important and can never be neglected.

\section{Acknowledgment}

This work was supported by JSPS KAKENHI (Grant Number JP17H0113). 


\section{References}

1) S. W. Lee, Y. Yang, H.-W. Lee, H. Ghasemi, D. Kraemer, G. Chen, Y. Cui, Nat. Commun. bf 5, 3942 (2013),

2) Y. Yang, S. W. Lee, H.-W. Lee, H. Ghasemi, J. Loomis, D. Kraemer, G. Zheng, Y. Cui, G. Chen, PNAS 111, 17011 (2014).

3) T. Shibata, Y. Fukuzumi, W. Kobayashi, and Y. Moritomo, Appl. Phys. Express 11, 017101 (2018).

4) Y. Fukuzumi, K. Amaha, H. Niwa, W. Kobayashi, and Y. Moritomo, Energy Technol. 10.1002/ente.201700952.

5) I. Quickenden and Y. Mua, J. Electrochem. Soc. 142, 3985 (1995).

6) J.-K. Park, Principle and Applications of Lithium Secondaray Battety (Wiley, Germany, 2012) P. 195.

7) David R.Lide (ed), CRC Handbook of Chemistry and Physics (CRC Press, Florida, 2003), 84th Edition. 